\title{
Shank-associated RH domain-interacting protein expression is upregulated in entodermal and mesodermal cancer or downregulated in ectodermal malignancy
}

\author{
YANHUA LIANG $^{1}$, BIAO CHEN ${ }^{1}$, FEN LIU $^{2}$, JIAMAN WANG $^{1}$, YAO YANG ${ }^{1}$, YAN ZHENG $^{1}$ and SHICUI TAN $^{1}$ \\ ${ }^{1}$ Department of Dermatology, Cosmetology and Venereology, Shenzhen Hospital, Southern Medical University, Shenzhen, \\ Guangdong 518101; ${ }^{2}$ Department of Histology and Embryology, Institute of Neuroscience, Wenzhou Medical University, \\ Wenzhou, Zhejiang 325035, P.R. China
}

Received November 23, 2017; Accepted September 17, 2018

DOI: $10.3892 / \mathrm{ol} .2018 .9514$

\begin{abstract}
Shank-associated RH domain-interacting protein (SHARPIN) is a type of linear ubiquitin chain-associated protein, which serves an important role in cell proliferation, apoptosis, organ development, immune and inflammatory reaction, initiation and development of malignant tumors. To evaluate SHARPIN expression in multiple malignant tumors derived from different germ layers, 14 types of cancer and their corresponding normal tissues were examined. Immunohistochemistry was performed to semi-quantify SHARPIN expression in multiple malignant tumors, and immunofluorescence was performed to evaluate the subcellular localization of SHARPIN in various malignant tumors. All the recruited cancer and paracancer samples originated from entoderm and mesoderm showed an upregulated expression of SHARPIN, whereas the cancer types that originated from ectoderm exhibited a downregulated or loss of SHARPIN expression. SHARPIN was primarily localized in the cytoplasm of cells and exhibited a faint signal in the nucleus, with the exception for lung cancer and esophagus cancer, in which malignant cells had aberrantly large nuclei and limited cytoplasm, which produced a signal in the nucleus but not in the cytoplasm. Conclusively, SHARPIN expression was upregulated in entodermal and mesodermal cancer types, but downregulated in ectodermal cancer types, indicating SHARPIN could act as either oncogene or anti-oncogene in malignant tumors derived from different germ layers.
\end{abstract}

Correspondence to: Professor Yanhua Liang, Department of Dermatology, Cosmetology and Venereology, Shenzhen Hospital, Southern Medical University, 1333 Xinhu Road, Shenzhen, Guangdong 518101, P.R. China

E-mail: liangdoctor@163.com

Key words: Shank-associated RH domain-interacting protein, malignant tumor, germ layer, expression

\section{Introduction}

Study of malignant tumor has always been a focus for the concern of human health because of their high morbidity and mortality. It was estimated that 5.3 million men and 4.7 million women would develop a malignant tumor annually and 6.2 million would die from the disease by the World Cancer Report 2014 of WHO. In 2014, approximately 14.1 million people were expected to develop cancer (1). Although malignant tumor mainly involves in the elderly, the morbidity and mortality of children with the disease present a rising trend. It has been the second cause of death in children merely much less than accidental emergency (2).

The etiology and pathogenesis of malignant tumor remain unclear, yet most of them have been related with accumulation of relative gene mutation or aberrant expression of gene. Shank-associated RH domain-interacting protein (SHARPIN) was firstly found as a scaffolding partner for Shank proteins. The Shank family of proteins highly expresses in postsynaptic density of excitatory synapses in brain. There are multiple domains of Shank for protein-protein interactions including proline-rich region, SAM domain, PDZ domain, SH3 domain and ankyrin domain. The Shank family is composed of three members: Shank1, Shank2 and Shank3. SHARPIN interacts with Shank through the ankyrin repeat domain of Shank1, which plays an important role in the formation and maintenance of excitatory synaptic structure. The succedent studies have shown that SHARPIN expresses in various organs relatively abundant including heart, brain and testis besides postsynaptic density, and localizes in the membrane and nuclei of cells (3), indicating that SHARPIN may play some roles in physiology and pathology process except for functioning as a scaffolding partner of Shank1. In 1993, study by Hogenesch et al showed that the phenotype of chronic proliferative dermatitis mutant $(c p d m)$ presented as chronic progressive dermatitis, absent Peyer's patches, abnormal structure of lymph node and spleen, immune dysfunction, and eosinophilic inflammation in multiple organs (4). In 2007, study by Seymour et al found that the genetic foundation of $c p d m$ phenotype derived from spontaneous mutation in the mouse Sharpin gene, suggesting that Sharpin may participate in cell proliferation, apoptosis, 
organ development, immune and inflammatory reaction (5). There were also evidences showed that increased expression of SHARPIN may involve in initiation and development of malignant tumor (6).

Our study widely explored the feature of SHARPIN expression in multiple malignant tumors originated from different germ layers by immunohistochemistry and immunofluorescence, confirmed the previous findings about SHARPIN's upregulation in entodermal and mesodermal cancers, and identified SHARPIN's downregulation, loss of function and translocation in ectodermal cancers, offering its complicated characters as oncogene or anti-oncogene.

\section{Materials and methods}

Materials. The study was conducted with the approval of the Institutional Review Board and Ethics Committee of Shenzhen Hospital, Southern Medical University (Shenzhen, China) and in accordance with the Declaration of Helsinki. Informed consent was obtained from all of the patients.

Samples of malignant tumors and their corresponding visceral organ tissues were obtained from the tissue bank of Shenzhen Hospital, Southern Medical University. Normal skin specimens were collected from the patients undergoing surgery at the plastic and constructive surgery department of Shenzhen Hospital, Southern Medical University. Malignant tumors were recruited as follows: Six kinds of malignant entodermal tumors including intrahepatic cholangiocellular carcinoma (ICC) $(\mathrm{N}=6)$, hepatocellular carcinoma (HCC) $(\mathrm{N}=5)$, lung cancer $(\mathrm{N}=6)$, esophageal cancer $(\mathrm{N}=6)$, laryngocarcinoma $(\mathrm{N}=5)$ and pancreatic cancer $(\mathrm{N}=7)$. Three kinds of malignant mesodermal tumors including breast cancer $(\mathrm{N}=10)$, endometrial cancer $(\mathrm{N}=4)$ and chromophobe renal cell carcinoma (CRCC) $(\mathrm{N}=4)$. Five kinds of malignant ectodermal tumors including basal cell carcinoma (BCC) $(\mathrm{N}=7)$, squamous cell carcinoma ( $\mathrm{SCC})(\mathrm{N}=5)$, Paget's disease $(\mathrm{N}=8)$, melanomas $(\mathrm{N}=7)$ and mycosis fungoides $(\mathrm{MF})(\mathrm{N}=3)$.

All samples were evaluated by two pathologists with a standard microscopic technique. Each case from the same block was stained with hematoxylin and eosin simultaneously for confirmation of the histologic diagnosis and tissue morphology and integrity.

Immunohistochemistry. Immunohistochemistry was performed with paraffin-embedded tissue sections from the above malignant tumors and their corresponding normal tissues. The procedure of immunohistochemistry was done according to the manufacturer's instructions. Briefly, immunostaining were implemented with anti-SHARPIN antibody (Santa Cruz Biotechnology Inc., Dallas, TX, USA) at $4^{\circ} \mathrm{C}$ using Histostain ${ }^{\mathrm{TM}}$-SP kits (OriGene Technologies, Inc., Beijing, China). After deparaffinization and hydration, antigen retrieval was carried out in a pressure cooker using $10 \mathrm{mM}$ sodium citrate buffer ( $\mathrm{pH} \mathrm{6.0)}$ ) at full power for $5 \mathrm{~min}$, and then the tissue sections were treated with $3 \%$ hydrogen peroxide for $15 \mathrm{~min}$, followed by treating with normal goat serum for $15 \mathrm{~min}$. The primary antibody was diluted (1:400) with primary antibody dilution buffer (Beyotime Institute of Biotechnology, Shanghai, China) and incubated with tissue sections for overnight at $4^{\circ} \mathrm{C}$. Then the slides were incubated with biotinylated goat anti rabbit $\mathrm{IgG}$ for $20 \mathrm{~min}$ and biotinylated horseradish peroxidase for $30 \mathrm{~min}$ and treated with 3,3-diaminobenzidine for 3 min sequentially, followed by being counterstained with Meyer's hematoxylin and mounted. Careful rinses were performed in every step using phosphate-buffered saline buffer (PBS) 3 times each of 5 min. Primary antibody dilution buffer incubated sample was used to be a negative control, and normal skin specimen incubated with anti-SHARPIN antibody (Santa Cruz Biotechnology Inc.) was used to be a positive control.

Immunofluorescence. The process of immunofluorescence was performed in accordance with the manufacturer's specifications. Deparaffinization, hydration and antigen retrieval of paraffin-embedded tissue sections were performed as immunohistochemistry. Subsequently, the tissue sections were rinsed for 3 times each of 5 min using PBS, then blocked by immunology staining blocking buffer (Beyotime Institute of Biotechnology) for $60 \mathrm{~min}$. The anti-SHARPIN antibody (BD Biosciences, Franklin Lakes, NJ, USA) was diluted (1:400) with PBS (Beyotime Institute of Biotechnology) and incubated for overnight at $4^{\circ} \mathrm{C}$ after decanting immunology staining blocking buffer. Decanting the primary antibody and rinsed for 3 times were as above described, tissue sections were treated with Immunol Fluorence Staining kit with Alexa Fluor 488-Labeled Goat Anti-Rabbit IgG (Beyotime Institute of Biotechnology) at room temperature for $1 \mathrm{~h}$ at dark, and then stained with $300 \mathrm{nM}$ 4',6-diamidino-2-phenyindole (DAPI; Leagene, Beijing, China) for $15 \mathrm{~min}$ and mounted on glass slides using Anti fade Mounting Medium (Beyotime Institute of Biotechnology). Imaging was processed with Olympus BX51 (Olympus, Corp., Tokyo, Japan).

Histologic scoring and statistical analysis. Each sample was scored by two pathologists blindly. SHARPIN protein was stained and assessed in tumors and their corresponding normal organ tissues. Each sample of tumor and the corresponding normal tissue was assessed using the cross-product (H score) (7), that is counting the percentage of sample cells staining at each of four staining intensities: 0 means no staining, 1 represents faint yellow, 2 is on behalf of deep yellow, 3 shows brown meaning a strong positive stain. For instance, one tumor sample staining at 2 of $60 \%$ tumor cells and 3 of $40 \%$ tumor cells, a combined $\mathrm{H}$ score is $[(60 \times 2)+(40 \times 3)]=240$ out of maximum of 300 . Scores from both pathologists showed a good correlation in which $85 \%$ of all the samples exhibited agreement within a range of 40 points. Samples in which a discrepancy of $>50$ points in scoring were reassessed and examined using the same standard microscope. The average of scores from both pathologists was used as the final $\mathrm{H}$ scores.

Data analyses were evaluated with IBM SPSS Statistics 23 (IBM Corp., Armonk, NY, USA), and values were expressed as mean $\pm \mathrm{SD}$ of 3 independent experiments. The significant differences between two or three groups were compared using Independent-Samples T Test or One-Way ANOVA, respectively. In Post Hoc Multiple Comparisons of One-Way ANOVA, S-N-K analysis was use when equal variances assumed, while Dunnett's T3 analysis was used when equal variances not assumed. $\mathrm{P}<0.05$ was considered to indicate a statistically significant difference. 

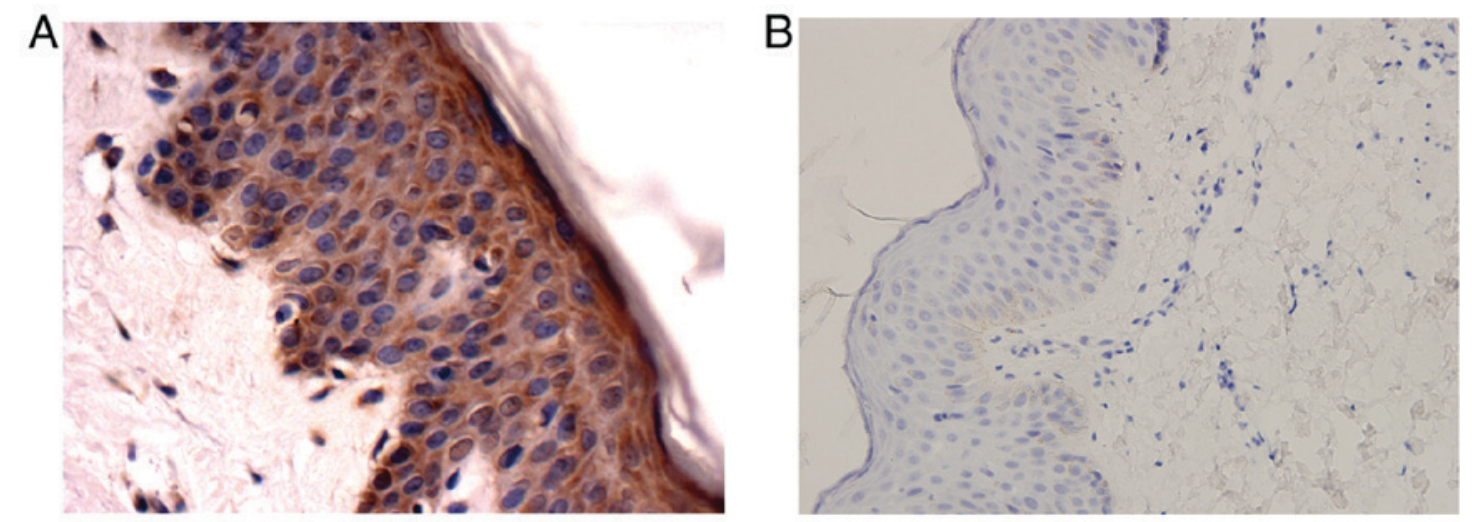

Figure 1. Verifying the specificity of anti-SHARPIN antibody. (A) Normal skin tissue section treated with anti-SHARPIN antibody was positively stained; (B) normal skin tissue section treated with primary antibody dilution buffer indicated no stain (A and B, original magnification, x400). SHARPIN, Shank-associated RH domain-interacting protein.

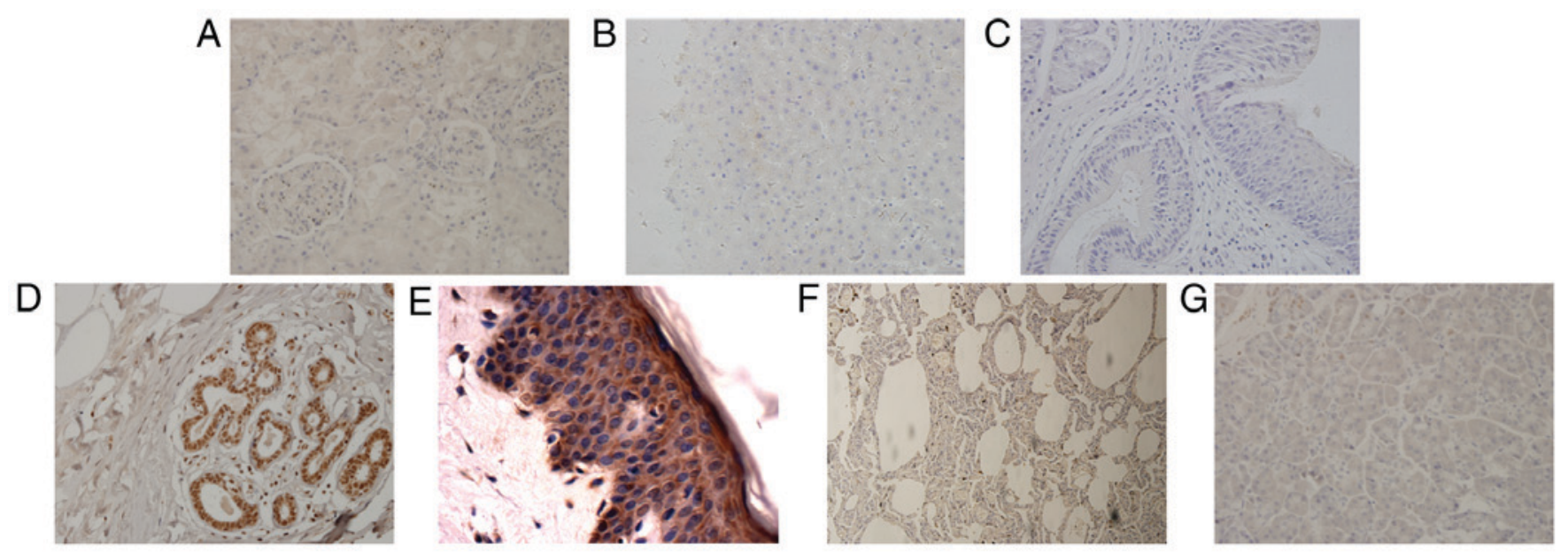

Figure 2. SHARPIN expression in normal organ tissues. IHC analysis demonstrated a weak stain in the kidney (A), liver (B) and (C) larynx. IHC analysis indicated a strong stain in the breast duct of the normal (D) breast tissue and (E) skin but moderate staining in (F) lung and (G) pancreas (A-G, original magnification, x400). SHARPIN, Shank-associated RH domain-interacting protein.

\section{Results}

In order to assess SHARPIN expression in multiple malignant tumors and their corresponding normal tissues, immunohistochemistry was carried out with anti-SHARPIN antibody. We checked the specificity of the antibody firstly with normal skin tissue. As mentioned above, sample treated with primary antibody dilution buffer served as a negative control while sample treated with anti-SHARPIN antibody served as a positive control. Compared with the negative control which showed no stain, the positive control exhibited a strong positive signal (Fig. 1). Then various malignant tumors and their corresponding paracancers and/or normal tissues were carried out immunohistochemistry to evaluate SHARPIN expression. H score was used to assess SHARPIN expression in cancer, paracancer and the corresponding normal tissue.

All the recruited normal organ tissues exhibited positive signal in which liver, kidney and larynx showed a faint stain. SHARPIN showed a strongest signal in both of the normal skin tissue and breast duct, and moderate signal in other tissues (Fig. 2).
SHARPIN expression in entodermal cancers. Six kinds of malignant tumors originated from entoderm, including ICC, HCC, lung cancer, esophageal cancer, laryngocarcinoma and pancreatic cancer, showed an elevated expression of SHARPIN (Fig. 3). Among those kinds of tumors, there are no reports about SHARPIN expression in ICC and laryngocarcinoma by now, and SHARPIN expression in HCC and pancreatic cancer is in accordance with previous findings (6). However, results of lung cancer and esophageal cancer do not accord with previous study which showed no difference of SHARPIN mRNA expression between cancer and normal tissue (6). Our explanation is that the SHARPIN mRNA in cancer tissue may be over translated.

SHARPIN expression in mesodermal cancers. Three kinds of malignant tumors originated from mesoderm, including breast cancer, endometrial cancer, CRCC, exhibited an upregulated expression of SHARPIN (Fig. 4). Our experimental results about breast cancer are in accordance with previous study $(8,9)$, and provided the first immunohistochemistrical findings in endometrial cancer and CRCC. Former studies also described enhanced SHARPIN expression in prostate cancer, 

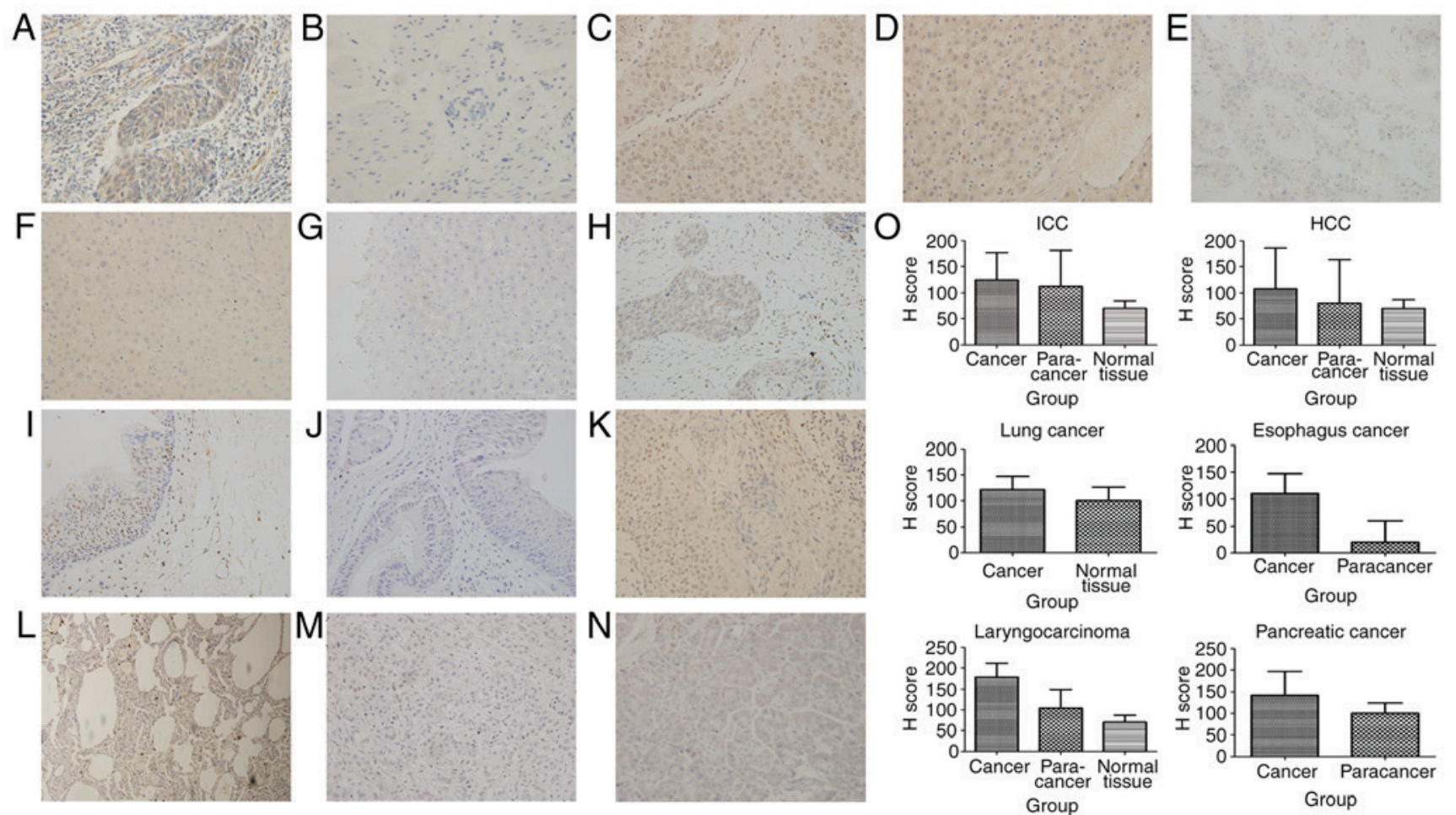

Figure 3. Tumors derived from entoderm exhibit upregulated expression of SHARPIN compared with their corresponding normal organ tissues. (A) Esophageal cancer, (B) paracancer of esophageal cancer (C) HCC, (D) paracancer of HCC, (E) ICC, (F) paracancer of ICC, (G) normal liver, (H) laryngocarcinoma, (I) paracancer of laryngocarcinoma, (J) normal larynx, (K) lung cancer, (L) normal lung, (M) pancreatic cancer, (N) normal pancreas, (O) H score of malignant tumors, paracancers and normal tissues derived from entoderm which are expressed in a histogram (A-N, original magnification, x400). SHARPIN, Shank-associated RH domain-interacting protein; HCC, hepatocellular carcinoma; ICC, cholangiocellular carcinoma.

renal clear cell adenoma and papillary serous adenocarcinoma of ovary which also originate from mesoderm (6), but were not included in our sample pool.

SHARPIN expression in ectodermal cancers. Five kinds of malignant tumors originated from ectoderm, including BCC, SCC, Paget's disease, melanomas and MF, showed a decreased expression of SHARPIN (Fig. 5). No investigations about SHARPIN's expression in ectodermal cancers were published.

Independent-Samples T Test of H score showed significant statistic difference of SHARPIN expression between some cancer nest and their corresponding paracancer or normal tissue, in which those tumors included MF, SCC, Paget's disease, BCC, melanomas, esophageal cancer. One-Way ANOVA analysis of $\mathrm{H}$ score showed significant statistic difference of SHARPIN expression among breast cancer, paracancer of breast cancer and normal breast tissue, in which SHARPIN expression in breast cancer was higher than paracancer of breast cancer, while SHARPIN expression in paracancer of breast cancer was higher than normal breast tissue.

Subcellular location of SHARPIN in cancers. Immunofluorescence was performed to confirm SHARPIN expression and evaluate the subcellular localization of SHARPIN in various malignant tumors and their corresponding normal tissues. For those recruited normal tissues, SHARPIN mainly expressed in the cytoplasm of cells and showed only a faint or no stain in the nucleus except for the normal lung tissue which exhibited a positive stain in the nucleus but not in the cytoplasm (Fig. 6). For those different kinds of malignant tumors, tumors in which SHARPIN expressed mainly in the cytoplasm but not nucleus or only a faint signal in the nucleus included ICC, HCC, laryngocarcinoma, pancreatic cancer, endometrial cancer, CRCC, SCC, Paget's disease, melanomas and MF (Fig. 7). However, tumors in which SHARPIN mainly showed positive signal in the nucleus but not or only weak signal in the cytoplasm included lung cancer, esophagus cancer (Fig. 8). Malignant tumors in which SHARPIN expressed in both of cytoplasm and nucleus but mainly in the cytoplasm included breast cancer and BCC (Fig. 9).

\section{Discussion}

All of human organs and tissues stem from embryo. Entoderm, mesoderm and ectoderm take shape successively by the third week of embryonic development. From the fourth week to the eighth week, those germ layers have differentiated into their corresponding tissues and organ anlage respectively. Among those germ layers, ectoderm differentiates into central nervous system, peripheral nervous system, epidermis and appendage of skin, breast, retina, crystalline lens, inner ear and olfactory epithelium, etc. Mesoderm differentiates into motor system including bone, cartilage and skeletal muscle, dermis and subcutaneous connective tissue of skin, most of urinary system and genital system, heart, blood vessel and lymphatic, etc. Entoderm differentiates into liver, pancreas, digestive glands of digestive tube, larynx, trachea, bronchus and lung. On the other side, the epithelium of some organs may originate from entoderm or ectoderm, however, the rest tissues of those organs 

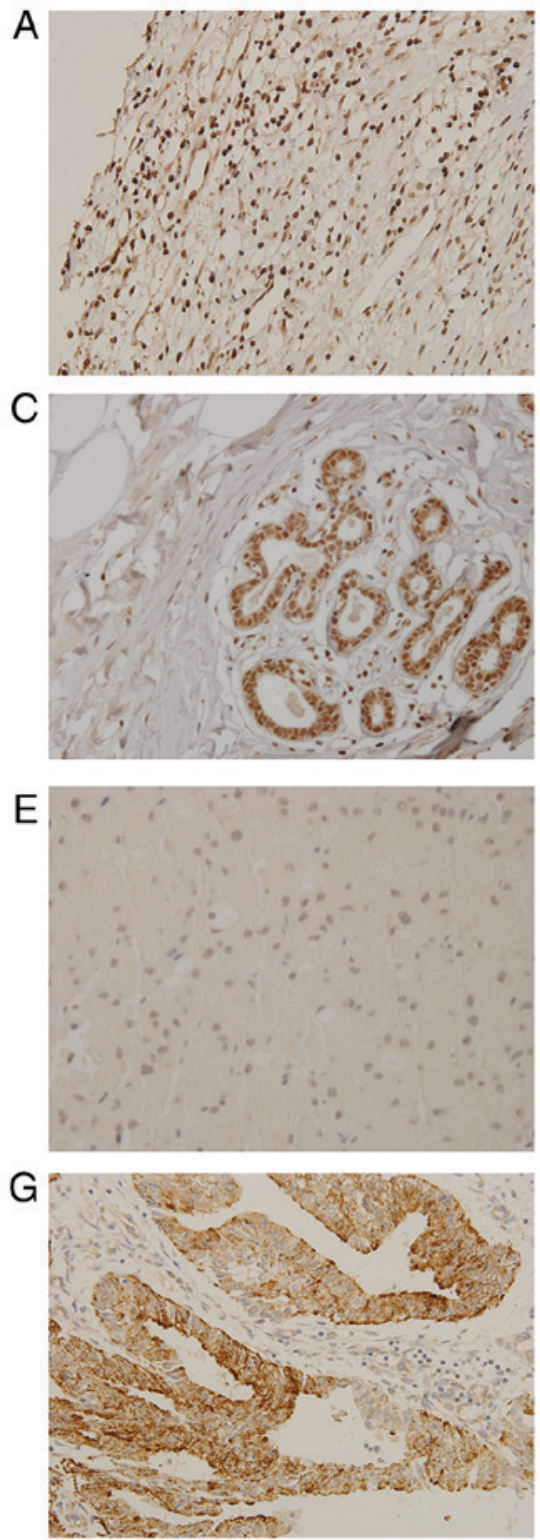

B

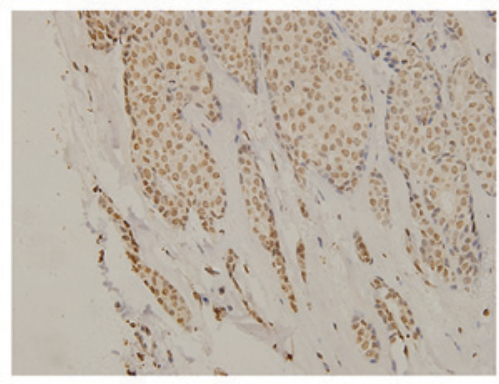

D

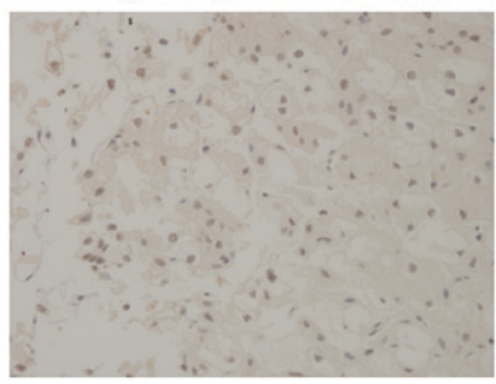

$\mathrm{F}$

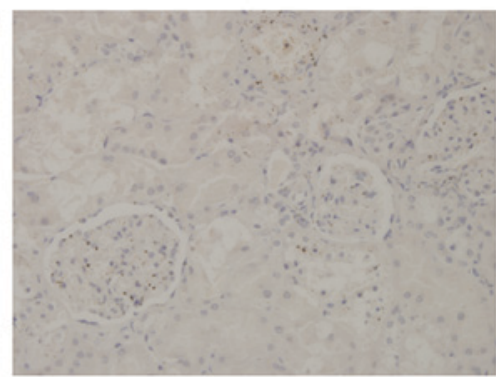

$\mathrm{H}$

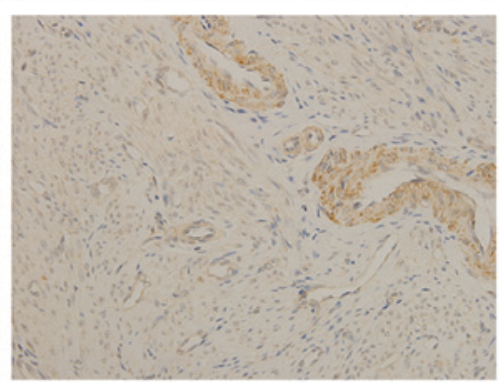

I

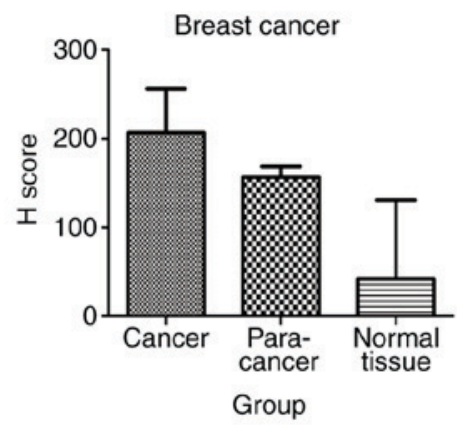

Endometrial cancer
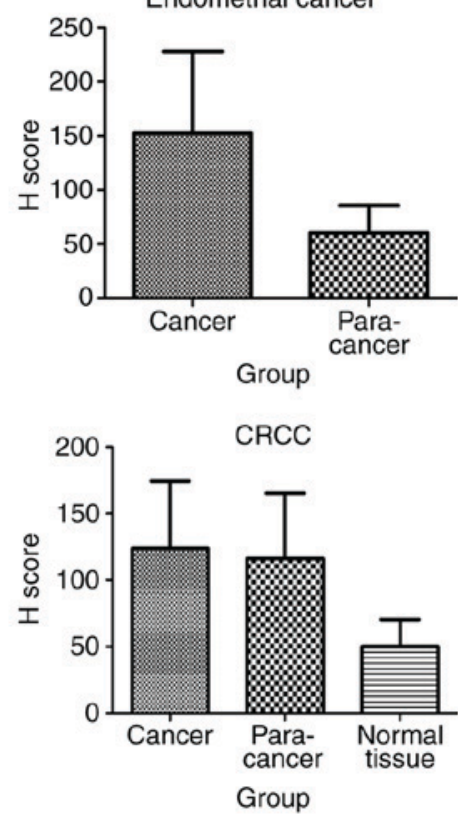

Figure 4. Tumors derived from mesoderm exhibit upregulated expression of SHARPIN compared with their corresponding normal organ tissues. (A) Breast cancer, (B) paracancer of breast cancer, (C) normal breast tissue, (D) CRCC, (E) paracancer of CRCC, (F) normal kidney; (G) endometrial cancer, (H) paracancer of endometrial cancer, (I) $\mathrm{H}$ score of malignant tumors, paracancers and normal tissues derived from mesoderm which are expressed in a histogram. (A-H, original magnification, x400). SHARPIN, Shank-associated RH domain-interacting protein; CRCC, chromophobe renal cell carcinoma.

derive from mesoderm, e.g., the skin of breast originates from ectoderm while the rest part derives from mesoderm. So breast cancer is categorized into tumor from mesoderm, except for mammary Paget's disease affecting breast duct, nipple and mammary areola which is not included in our experiment. All of the recruited cases of Paget's disease are extramammary Paget's disease from ectoderm mainly affecting on scrotum, perineum, crissum or axilla.

SHARPIN is a kind of linear ubiquitin chain related protein which has multiple functions. Recent studies have indicated that SHARPIN can induce cell survival via activating NF- $\kappa \mathrm{B}$ signaling pathway in hepatocytes (10), epithelial cells (11), and even osteosarcoma cells (12). In addition, SHARPIN can modulate keratinocytes apoptosis mediated by mitochondria (13). SHARPIN also involves in tumorigenesis and tumor progression reported by recent studies. After analysis of expression and function between SHARPIN and PTEN in 2010,
He et al considered that SHARPIN affects tumorigenesis via inhibition of PTEN function (14). As a tumor suppressor, PTEN dephosphorylates phosphotidylinositol-3,4,5-triphosphate (PIP3) at the plasma membrane, and in the nucleus it regulates genome stability (15). PTEN can be inactivated by PTEN negative regulators (PTEN-NRs). As a PTEN-NRs, shank-interacting protein-like 1 (SIPL1), namely SHARPIN, can interact with PTEN via its UBL domain, resulting in inhibition of the PIP3 phosphatase activity of PTEN. SIPL1 inhibits function of PTEN in PTEN-positive human primary cervical cancer tissue. Knockdown of SIPL1 expression by siRNA inhibits the growth of both human prostate carcinoma cells DU145 and HeLa cells in vitro and in vivo in axenograft tumor model, and upregulated expression of SIPL1 protects human U87 glioma cells from growth inhibition induced by PTEN (14). In 2015 Bii identified SHARPIN as a breast cancer metastasis gene and prognostic biomarker by a novel gamma retroviral shuttle vector 

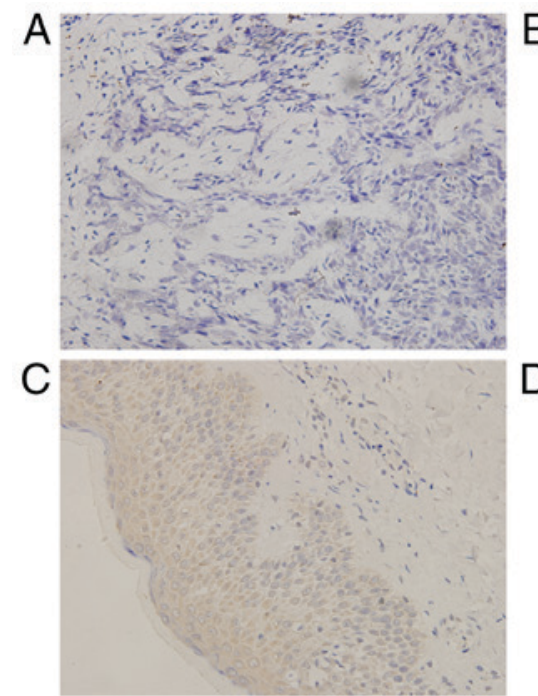

E

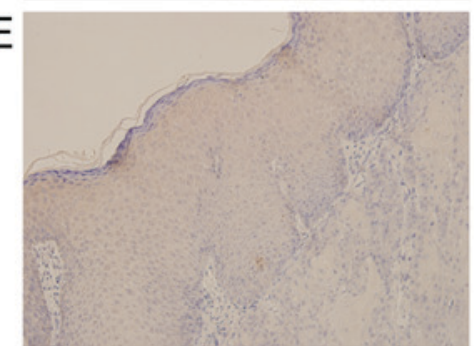

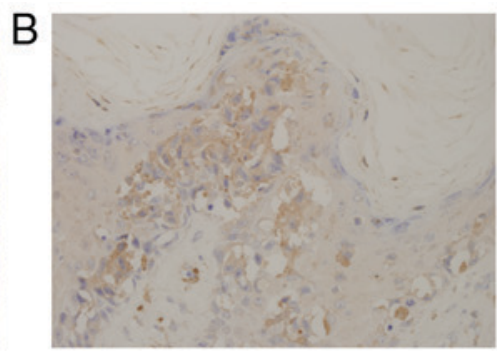

D
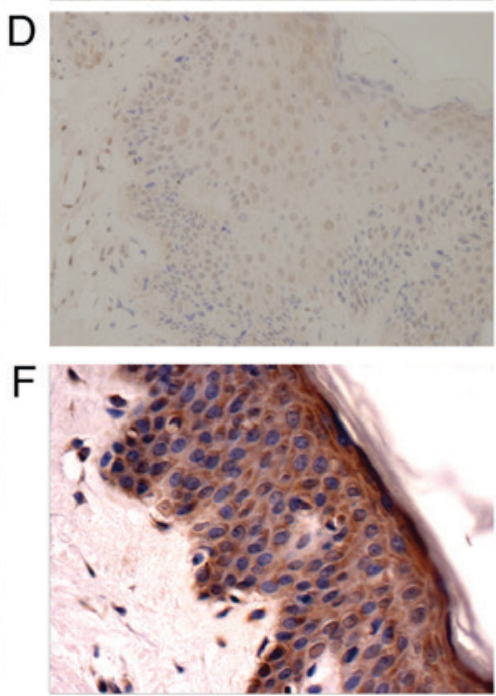

G
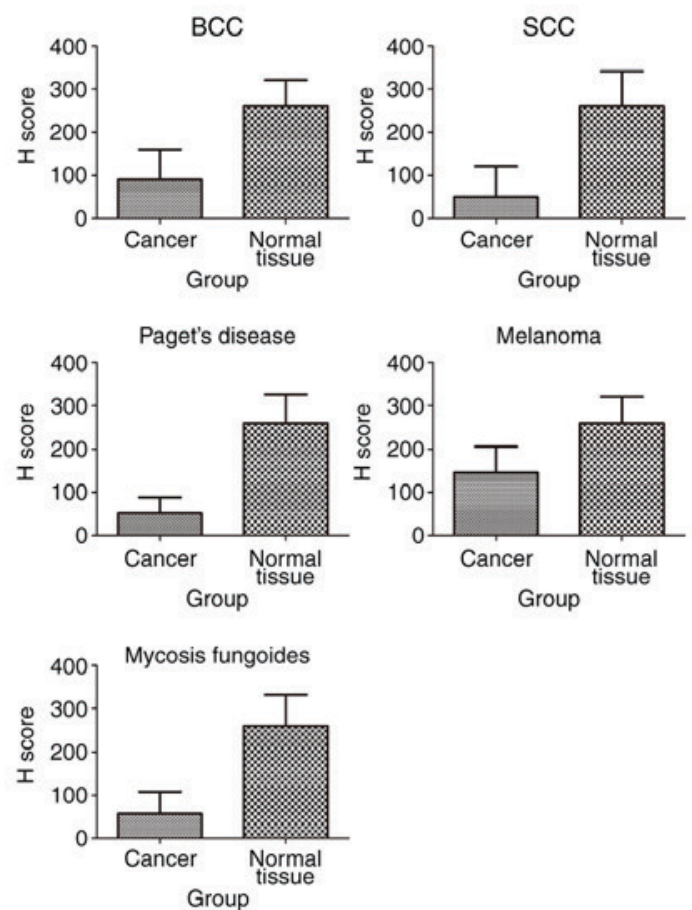

Figure 5. Tumors derived from ectoderm exhibit downregulated expression of SHARPIN compared with their corresponding normal tissues. (A) BCC, (B) melanoma, (C) mycosis fungoides, (D) Paget's disease, (E) SCC and (F) normal skin. (G) H score of malignant tumors and normal tissues derived from ectoderm which are expressed in a histogram; respectively (A-F, original magnification, x400). SHARPIN, Shank-associated RH domain-interacting protein; BCC, basal cell carcinoma; SCC, squamous cell carcinoma.
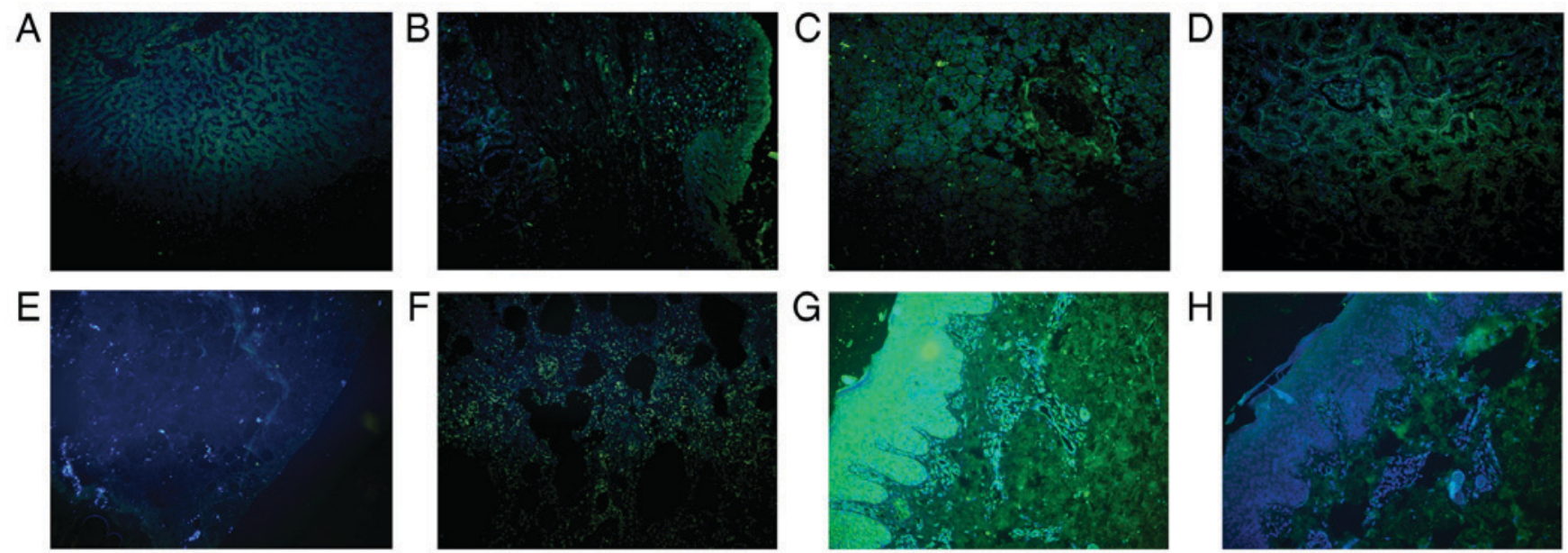

Figure 6. SHARPIN is mainly expressed in the cytoplasm of cells in the normal tissues recruited, with the exception for the normal lung tissue. (A) Liver, (B) larynx, (C) pancreas, (D) kidney, (E) breast, (F) lung samples. (G) Formalin fixed and paraffin embedded normal skin sample incubated with anti-SHARPIN antibody and $(\mathrm{H})$ normal skin sample incubated with PBS, which acted as the imunofluorescence negative control. Blue and green staining in figure of immunofluorescence indicate nucleus staining and SHARPIN positive staining of cells, respectively (A-H, original magnification, x200). SHARPIN, Shank-associated RH domain-interacting protein; PBS, phosphate-buffered saline buffer.

insertional mutagenesis screen (8). Study by De Melo and Tang described a positive correlation of SHARPIN with breast cancer tumorigenesis (9). In 2010, Jung et al analyzed genome-wide differences in gene expression in 11 kinds of visceral malignant tumors originated from breast, colon, kidney, liver, lung, esophagus, ovary, pancreas, prostate, rectum, and stomach. Among of those malignant tumors, the expression of SHARPIN in renal clear cell adenoma, HCC, papillary serous adenocarcinoma of ovary and pancreas adenocarcinoma increases, experiment in vitro identified that overexpression of SHARPIN is related with tumorigenesis (6). Consequently, it is postulated that SHARPIN potentially involves in the development and proliferation of cells, overexpression of SHARPIN may closely promote the initiation and development of malignant tumor. 


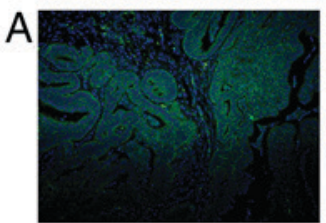

F
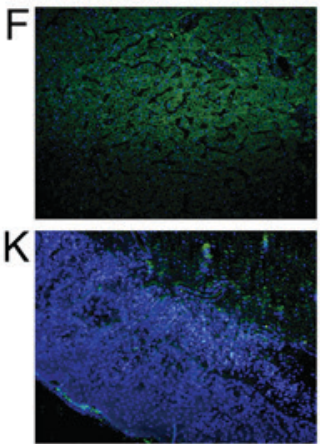

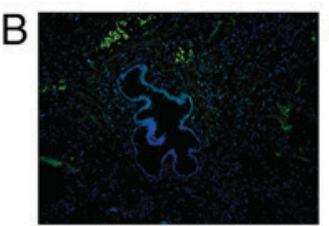

G
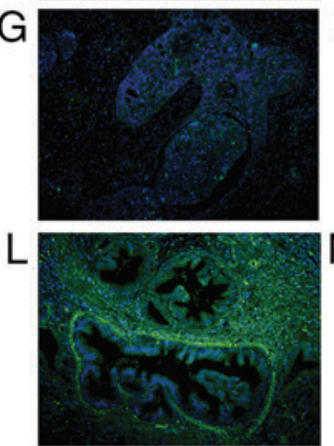

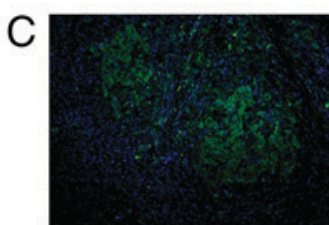

$\mathrm{H}$
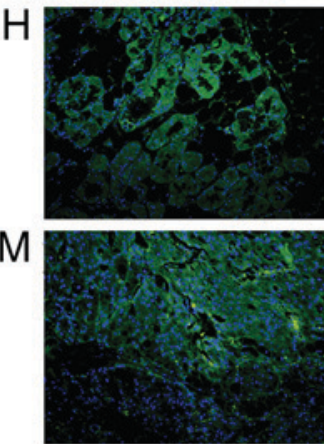
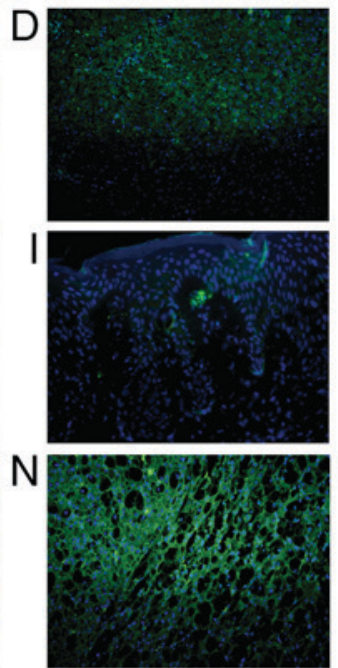

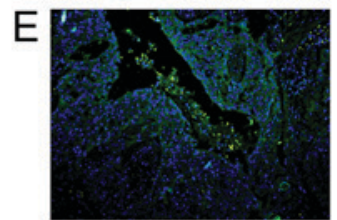

$J$
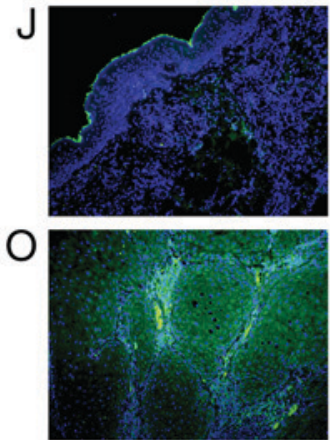

Figure 7. Subcellular location of SHARPIN in different cancer types in which SHARPIN mainly localizes in the cytoplasm of malignant cells. Endometrial cancer, HCC, ICC, laryngocarcinoma, melanomas, mycosis fungoides, Paget's disease, pancreatic cancer, CRCC and SCC were examined. (A) Endometrial cancer and (B) paracancer of endometrial cancer; (C) HCC and (D) paracancer of HCC; (E) ICC and (F) paracancer of ICC; (G) laryngocarcinoma and (H) paracancer of laryngocarcinoma; (I) melanomas; (J) mycosis fungoides; (K) Paget's disease; (L) pancreatic cancer; (M) CRCC and (N) paracancer of CRCC; and (O) SCC. Blue and green staining in figure of immunofluorescence indicate nucleus staining and SHARPIN positive staining of cells, respectively (A-O, original magnification, x200). SHARPIN, Shank-associated RH domain-interacting protein; HCC, hepatocellular carcinoma; ICC, cholangiocellular carcinoma; BCC, basal cell carcinoma; SCC, squamous cell carcinoma.
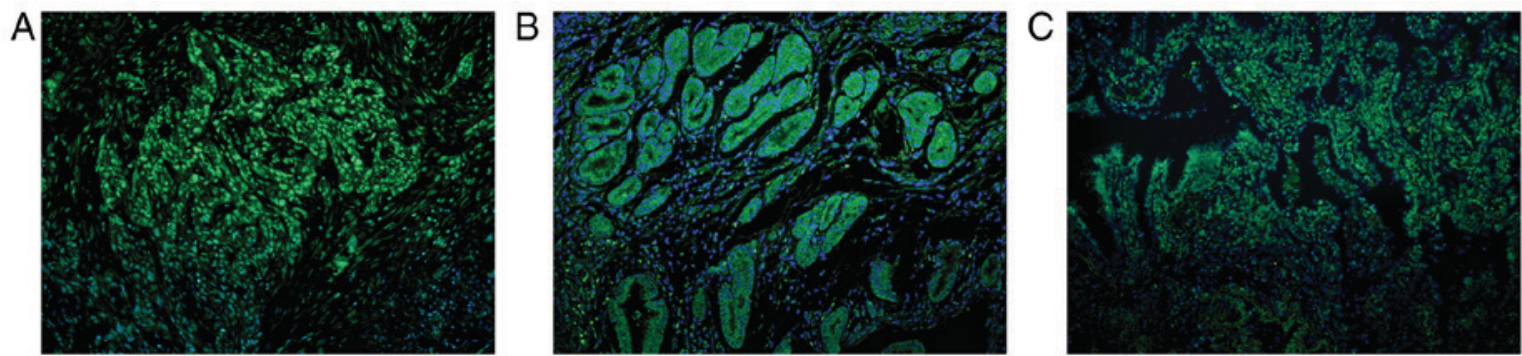

Figure 8. SHARPIN mainly localizes in the nucleus of malignant cells in tumors, including esophagus cancer and lung cancer. (A) Esophageal cancer (B) paracancer of esophageal cancer and (C) lung cancer. Blue and green staining in figure of immunofluorescence indicate nucleus staining and SHARPIN positive staining of cells, respectively (A-C, original magnification, x200). SHARPIN, Shank-associated RH domain-interacting protein.
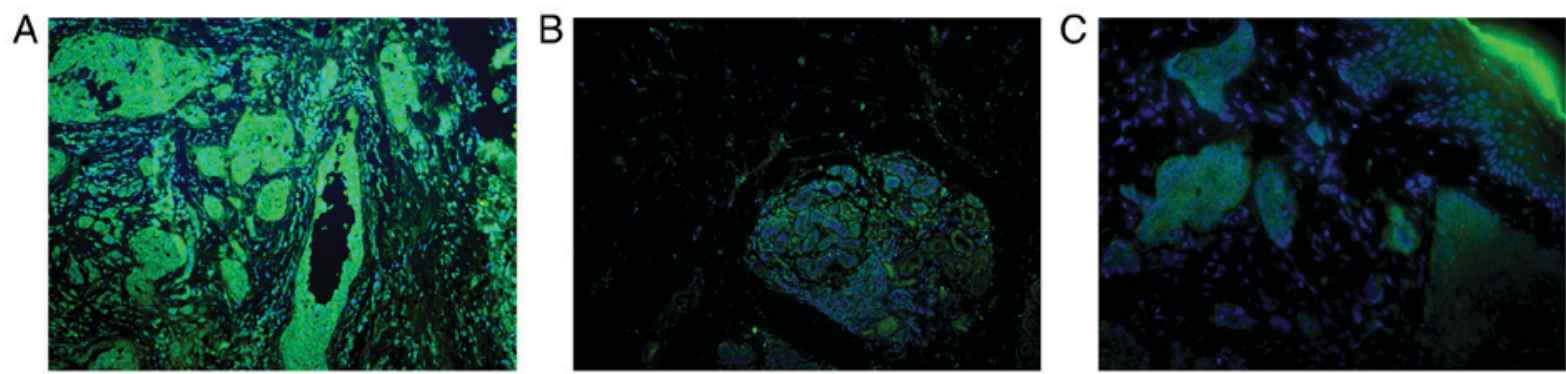

Figure 9. SHARPIN localizes in the cytoplasm and nucleus of malignant cells but mainly in cytoplasm in tumors, including breast cancer and BCC. (A) Breast cancer, (B) paracancer of breast cancer and (C) BCC. Blue and green staining in figure of immunofluorescence indicate nucleus staining and SHARPIN positive staining of cells, respectively (A-C, original magnification, x200). SHARPIN, Shank-associated RH domain-interacting protein; BCC, basal cell carcinoma.

Immunoblot analysis revealed that SHARPIN protein relatively highly expresses in lung, brain and spleen, and expresses at a lower level in testis, kidney, skeletal muscle, liver and heart (3). In our study, 7 kinds of normal tissues including liver, lung, larynx, pancreas, breast, kidney and skin exhibited positive signal, indicating that SHARPIN widely expresses in human normal tissue and maybe serve as a multiple functional protein more than an interactor of Shank proteins. Those results and enhanced expression of SHARPIN in breast cancer, HCC and pancreatic cancer showed in our study are in accordance with the previous study which confirmed the repeatability and the results' reliability of our work. Our study 
also showed that all of the recruited tumors and paracancer samples originated from entoderm and mesoderm (including prostate cancer, renal clear cell adenoma and papillary serous adenocarcinoma of ovary from mesoderm which previous studies reported but not recruited in our study) showed an upregulated expression of SHARPIN, while tumor originated from ectoderm exhibited a downregulated expression. These wide-in-depth discoveries may suggest that SHARPIN serves as a promoting effect in the pathogenesis of tumors derived from entoderm and mesoderm, while plays a suppressor role in tumors derived from ectoderm. It is reasonable to reassess the role of SHARPIN in the initiation and development of malignant tumors. SHARPIN could play a different or even opposite role in malignant tumors derived from different germ layers.

Indeed, the function of a specific gene in different tissues may be different; it is spatial-temporal dependence even in a kind of cell to react appropriately to various changes of internal environment and external environment. For example, Zinc-fingers and homeoboxes 1 (ZHX1) is a transcription repressor which involves in pathogenesis of multiple human cancers; study by Kwon et al showed that the expression of ZHX1 increases in cholangiocarcinoma (CCA) tissues and ZHX1 promotes CCA cell proliferation, migration, and invasion, functioning as an oncogene in CCA (16); however, study by Ma et al exhibited that ZHX1 expression is downregulated in gastric cancer, ZHX1 could inhibit cell growth by inducing cell-cycle arrest and apoptosis, showing a role of tumor suppressor in gastric cancer (17).

In this study, there are five kinds of common skin tumors recruited from ectoderm including BCC, SCC, Paget's disease, melanomas and MF. Reduced expression of SHARPIN in those skin cancers is contrary to previous studies which enhanced expression of SHARPIN was observed in various of tumors such as prostate cancer, breast cancer and HCC. SHARPIN may function as a tumor suppressor in those skin cancers, which is a novel discovery of SHARPIN function. Reduced SHARPIN expression in part of malignant cells and loss expression of SHARPIN in the other part of malignant cells were observed in these skin cancers.

Primary hepatic carcinoma can be divided into HCC, ICC and mixed hepatocellular carcinoma by histological differentiation. HCC occurs in hepatocytes while ICC occurs in intrahepatic biliary epithelial cells. Previous study described elevated expression of SHARPIN in HCC, while enhanced SHARPIN expression was observed in both HCC and ICC of our study, indicating a pro-oncogenic role in the tumorigenesis of primary hepatic carcinoma. Both of renal clear cell carcinoma (RCCC) and CRCC belong to renal cell carcinoma differing in the shape of malignant cells. RCCC account for approximately $70-80 \%$ and CRCC account for $5 \%$ of renal cell carcinoma. Former study exhibited increased SHARPIN expression in RCCC (6), while enhanced SHARPIN expression was also observed in CRCC in our study, suggesting that SHARPIN may function as a pro-oncogenic factor in the tumorigenesis of renal cell carcinoma.

Immunoblot analysis of adult rat brain revealed that SHARPIN protein widely expresses among subcellular fractions, with moderate amount in light membrane fractions and crude synaptosomal, a large amount in cytosolic fractions (3). There were also other studies showed that SHARPIN localizes in membranes and nuclei of cells $(6,18)$. To assess the subcellular localization of SHARPIN in various malignant tumors and their corresponding normal tissues, we conducted immunofluorescence analysis of SHARPIN expression in the above tissues. For normal tissues recruited, SHARPIN mainly localized in the cytoplasm of cells and showed no or only a faint signal in the nucleus except for the normal lung tissue which exhibited an opposite phenomenon. For these 14 kinds of malignant tumors recruited in this study, SHARPIN also mainly localized in the cytoplasm of cells and presented no or only a faint signal in the nucleus except for lung cancer and esophagus cancer, in which malignant cells have aberrantly big nucleus but basically no cytoplasm, exhibited signal in the nucleus of cells but not in the cytoplasm. It is postulated that SHARPIN mainly has a role in the cytoplasm of cells. Indeed, previous studies have shown that SHARPIN can interact with NF- $\kappa \mathrm{B}, \mathrm{PTEN}$, integrin and MAPK in the cytoplasm $(14,19,20)$. Also SHARPIN have a role in the nucleus, SHARPIN can combines with EYA1 and EYA2 (eyes absent homolog 1 and 2) directly, which enhances relative targeted gene expression in the development of several tissues (21). In our study, SHARPIN localized in the nucleus of malignant cells of tumors such as lung cancer and esophagus cancer, but its relationship with malignant tumor still remains unclear.

We conducted a wide-range preliminary screening research about SHARPIN expression in various cancers derived from different germ layers, verifying the previous studies about SHARPIN expression in 7 kinds of normal tissues and 3 kinds of tumors including HCC, breast cancer and pancreatic cancer, and identified that the SHARPIN expression pattern in ectodermal cancers is different from entodermal and mesodermal malignancies, indicating a dual role in tumorigenesis in which SHARPIN could function as a pro-oncogenic role in entoderm and mesoderm or a tumor suppression factor in ectoderm. However, a limitation of the study is that no experiments were conducted to confirm the role of SHARPIN in the tumors, and further in vitro and in vivo study is ongoing to investigate the role of SHARPIN in skin malignancies.

\section{Acknowledgements}

Not applicable.

\section{Funding}

The present research was supported by a grant from National Natural Science Foundation of China (grant no. 81371724).

\section{Availability of data and materials}

The datasets used and/or analyzed during the current study are available from the corresponding author on reasonable request.

\section{Authors' contributions}

YL designed and guided this study, and outlined and revised the manuscript. BC performed the majority of the experiments and drafted the manuscript. FL, YY and YZ collected samples and assisted in the study design. JW conducted data analysis, 
data interpretation and generated the figures. ST performed data collection and the literature search. All authors read and approved the final manuscript.

\section{Ethics approval and consent to participate}

The study was conducted with the approval of the Institutional Review Board and Ethics Committee of Shenzhen Hospital, Southern Medical University and in accordance with the Declaration of Helsinki. Informed consent was obtained from all of the patients.

\section{Patient consent for publication}

The patients provided written informed consent for the publication of any associated data and accompanying images.

\section{Competing interests}

The authors declare that they have no competing interests.

\section{References}

1. McGuire S: World Cancer Report 2014. Geneva, Switzerland: World Health Organization, International Agency for Research on Cancer, WHO Press, 2015. Adv Nutr 7: 418-419, 2016.

2. Kaatsch P: Epidemiology of childhood cancer. Cancer Treat Rev 36: 277-285, 2010.

3. Lim S, Sala C, Yoon J, Park S, Kuroda S, Sheng M and Kim E: Sharpin, a novel postsynaptic density protein that directly interacts with the shank family of proteins. Mol Cell Neurosci 17 385-397, 2001

4. Hogenesch H, Gijbels MJ, Offerman E, van Hooft J, van Bekkum DW and Zurcher C: A spontaneous mutation characterized by chronic proliferative dermatitis in C57BL mice. Am J Pathol 143: 972-982, 1993.

5. Seymour RE, Hasham MG, Cox GA, Shultz LD, Hogenesch H, Roopenian DC and Sundberg JP: Spontaneous mutations in the mouse Sharpin gene result in multiorgan inflammation, immune system dysregulation and dermatitis. Genes Immun 8: 416-421, 2007.

6. Jung J, Kim JM, Park B, Cheon Y, Lee B, Choo SH, Koh SS and Lee S: Newly identified tumor-associated role of human Sharpin. Mol Cell Biochem 340: 161-167, 2010.
7. Bollag G, Hirth P, Tsai J, Zhang J, Ibrahim PN, Cho H, Spevak W, Zhang C, Zhang Y, Habets G, et al: Clinical efficacy of a RAF inhibitor needs broad target blockade in BRAF-mutant melanoma. Nature 467: 596-599, 2010.

8. Bii VM, Rae DT and Trobridge GD: A novel gammaretroviral shuttle vector insertional mutagenesis screen identifies SHARPIN as a breast cancer metastasis gene and prognostic biomarker. Oncotarget 6: 39507-39520, 2015.

9. De Melo J and Tang D: Elevation of SIPL1 (SHARPIN) increases breast cancer risk. PLoS One 10: e0127546, 2015.

10. Sieber S, Lange N, Kollmorgen G, Erhardt A, Quaas A, Gontarewicz A, Sass G, Tiegs G and Kreienkamp HJ: Sharpin contributes to $\mathrm{TNF} \alpha$ dependent $\mathrm{NF} \kappa \mathrm{B}$ activation and anti-apoptotic signalling in hepatocytes. PLoS One 7: e29993, 2012.

11. Gerlach B, Cordier SM, Schmukle AC, Emmerich CH, Rieser E, Haas TL, Webb AI, Rickard JA, Anderton H, Wong WW, et al: Linear ubiquitination prevents inflammation and regulates immune signalling. Nature 471: 591-596, 2011.

12. Tomonaga M, Hashimoto N, Tokunaga F, Onishi M, Myoui A, Yoshikawa $\mathrm{H}$ and Iwai K: Activation of nuclear factor-kappa B by linear ubiquitin chain assembly complex contributes to lung metastasis of osteosarcoma cells. Int J Oncol 40: 409-417, 2012.

13. Liang Y and Sundberg JP: SHARPIN regulates mitochondria-dependent apoptosis in keratinocytes. J Dermatol Sci 63: 148-153, 2011.

14. He L, Ingram A, Rybak AP and Tang D: Shank-interacting protein-like 1 promotes tumorigenesis via PTEN inhibition in human tumor cells. J Clin Invest 120: 2094-2108, 2010.

15. Shi Y, Paluch BE, Wang X and Jiang X: PTEN at a glance. J Cell Sci 125: 4687-4692, 2012.

16. Kwon RJ, Han ME, Kim JY, Liu L, Kim YH, Jung JS and Oh SO: ZHX1 promotes the proliferation, migration and invasion of cholangiocarcinoma cells. PLoS One 11: e0165516, 2016.

17. Ma X, Huang M, Wang Z, Liu B, Zhu Z and Li C: ZHX1 inhibits gastric cancer cell growth through inducing cell-cycle arrest and apoptosis. J Cancer 7: 60-68, 2016.

18. Wang Z, Potter CS, Sundberg JP and Hogenesch H: SHARPIN is a key regulator of immune and inflammatory responses. J Cell Mol Med 16: 2271-2279, 2012.

19. Tokunaga F, Nakagawa T, Nakahara M, Saeki Y, Taniguchi M, Sakata S, Tanaka K, Nakano H and Iwai K: SHARPIN is a component of the NF- $\kappa \mathrm{B}$-activating linear ubiquitin chain assembly complex. Nature 471: 633-636, 2011.

20. Rantala JK, Pouwels J, Pellinen T, Veltel S, Laasola P, Mattila E, Potter CS, Duffy T, Sundberg JP, Kallioniemi O, et al: SHARPIN is an endogenous inhibitor of $\beta 1$-integrin activation. Nat Cell Biol 13: 1315-1324, 2011.

21. Landgraf K, Bollig F, Trowe MO, Besenbeck B, Ebert C, Kruspe D, Kispert A, Hänel F and Englert C: Sipl1 and Rbck1 are novel Eya1-binding proteins with a role in craniofacial development. Mol Cell Biol 30: 5764-5775, 2010.

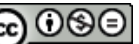

This work is licensed under a Creative Commons Attribution-NonCommercial-NoDerivatives 4.0 International (CC BY-NC-ND 4.0) License. 